\title{
EXISTENCE AND MULTIPLICITY OF SOLUTIONS FOR NONLINEAR SCHRÖDINGER-KIRCHHOFF-TYPE EQUATIONS
}

\author{
Haibo Chen, Hongliang Liu, and Liping Xu
}

ABSTRACT. In this paper, we consider the following Schrödinger-Kirchhoff-type equations

$$
\left[a+b\left(\int_{\mathbb{R}^{N}}\left(|\nabla u|^{2}+V(x)|u|^{2}\right) d x\right)\right][-\Delta u+V(x) u]=f(x, u), \text { in } \mathbb{R}^{N} .
$$

Under certain assumptions on $V$ and $f$, some new criteria on the existence and multiplicity of nontrivial solutions are established by the Morse theory with local linking and the genus properties in critical point theory. Some results from the previously literature are significantly extended and complemented.

\section{Introduction and main results}

In this paper, we investigate the existence and multiplicity of solutions to the following Schrödinger-Kirchhoff-type equations

$$
\text { (1.1) }\left[a+b\left(\int_{\mathbb{R}^{N}}\left(|\nabla u|^{2}+V(x)|u|^{2}\right) d x\right)\right][-\Delta u+V(x) u]=f(x, u) \text {, in } \mathbb{R}^{N},
$$

where $N \geq 2, a, b$ are positive constants, and the potential $V$ satisfies the following condition:

$$
V(x) \in C\left(\mathbb{R}^{N}, \mathbb{R}\right) \text { satisfies } \inf _{\mathbb{R}^{N}} V(x) \geq \alpha>0 .
$$

Problem (1.1) is related to the stationary analogue of the equation

$$
u_{t t}-\left(a+b \int_{\Omega}|\nabla u|^{2}\right) \triangle u=h(x, u),
$$

Received October 11, 2014.

2010 Mathematics Subject Classification. 35J20, 35J15, 35J60.

Key words and phrases. Schrödinger-Kirchhoff-type, Morse theory, critical groups, variational methods, genus.

Research supported by Natural Science Foundation of China 11271372, Hunan Provincial Natural Science Foundation of China 12JJ2004 and Hunan Provincial Foundation For Postgraduate CX2014B044. 
which was presented by Kirchhoff [10] to describe the transversal oscillations of a stretched string, where $u$ denotes the displacement, $h$ is the external force, $b$ represents the initial tension, and $a$ is related to the intrinsic properties of the string.

In recent years, the following Kirchhoff type problem

$$
-\left(a+b \int_{\mathbb{R}^{N}}|\nabla u|^{2} d x\right) \Delta u+\lambda V(x) u=f(x, u), \quad \text { in } \mathbb{R}^{N}
$$

has been widely studied by many authors since Lions [13] proposed an abstract framework. More precisely, $\mathrm{Wu}$ in [23] studied the existence of nontrivial solutions and infinitely many high energy solutions of problem (1.2) by using a symmetric mountain pass theorem. Liu and He in [14] also investigated the existence of infinitely many high energy solutions of problem (1.2) under superlinear case by variant version of fountain theorem. In [22], Sun and $\mathrm{Wu}$ investigated the existence and the non-existence of nontrivial solutions of problem (1.2) by using variational methods and explored the concentration of solutions. When $N=3, \mathrm{Li}$ and Ye considered problem (1.2) with pure power nonlinearities $f(x, u)=|u|^{p-1} u$ in $\mathbb{R}^{3}$. By using a monotonicity trick and a new version of global compactness lemma, they get that the problem has a positive ground state solution that the result can be viewed as a partial extension of [9] where the authors studied the existence and concentration behavior of positive solutions of problem (1.2). In addition, other interesting results on the related Kirchhoff equations can be found in [1, 6, 7, 8, 16, 18, 24].

It is worth mentioning that in [12] $\mathrm{Li}$ et al. studied the following autonomous Kirchhoff type problem

$$
\left(a+\lambda \int_{\mathbb{R}^{N}}|\nabla u|^{2}+\lambda b \int_{\mathbb{R}^{N}} u^{2} d x\right)[-\Delta u+b u]=f(u), \quad \text { in } \mathbb{R}^{N},
$$

where $N \geq 3, a, b$ are positive constants and $\lambda \geq 0$ is a parameter. They proved that problem (1.3) has at least one positive solution under the following condition:

$\left(H_{1}\right) f \in C(\mathbb{R}+, \mathbb{R}+)$ and $|f(u)| \leq C\left(|u|+|u|^{p-1}\right)$ for all $t \in \mathbb{R}+=[0, \infty)$ and some $p \in\left(2,2^{*}\right)$, where $2^{*}=2 N /(N-2)$ for $N \geq 3$.

Obviously, a question shows up that what will happen if $p \in(1,2)$. This is what we interested in the present paper. More precisely, the case we consider in the present paper is that $f(x, u)$ satisfies the following condition.

( f1) $f \in C\left(\mathbb{R}^{N} \times \mathbb{R}, \mathbb{R}\right)$, and there exist constants $1<\delta_{1}<\delta_{2}<\cdots<\delta_{m}<$ 2 , and functions $a_{i} \in L^{\frac{2}{2-\delta_{i}}}\left(\mathbb{R}^{N}, \mathbb{R}_{+}\right),(i=1,2, \ldots, m)$ such that

$$
|f(x, u)| \leq \sum_{i=1}^{m} \delta_{i} a_{i}(x)|u|^{\delta_{i}-1}
$$

The Morse theory and genus properties in critical point theory are the useful tools in looking for the solutions for the variational problem (see, for example [4, $5,15,17,21])$. However, to the best of our knowledge, there is no existed works 
dealing with problem (1.1) by combining Morse theory and genus properties up to now. Motivated by the above facts and the main purpose of this paper is to study the existence and multiplicity of nontrivial solutions of problem (1.1). The proofs are based on combining Morse theory with local linking method and the genus properties in critical point theory.

Now, we are ready to state the main results of this paper.

Theorem 1.1. Let the condition $(V)$ and $(f 1)$ be satisfied and the following condition holds.

( $f 2$ ) there exist an open set $\Omega \subset \mathbb{R}^{N}$ and three constants $\zeta, \eta>0, \kappa \in(1,2)$ such that

$$
F(x, u) \geq \eta|u|^{\kappa}, \quad \forall(x, u) \in \Omega \times[-\zeta, \zeta],
$$

where and in the sequel $F(x, u)=\int_{0}^{u} f(x, s) d s$.

Then problem (1.1) possesses at least one nontrivial solution.

Theorem 1.2. Let the condition $(V)$ and $(f 1)$ be satisfied and the following condition holds.

(f3) there exist $1<\tau<2,0<c_{1}<c_{2}<\frac{a}{\varsigma \gamma_{2}^{2}}$ such that

$$
c_{1}|u|^{\tau} \leq F(x, u) \leq c_{2}|u|^{2} \quad \text { for }|u| \text { small }
$$

where $\varsigma \geq 2, \gamma_{2}$ is Sobolev constant.

Then problem (1.1) possesses at least two nontrivial solutions.

Theorem 1.3. Let all the conditions in Theorem 1.1 be satisfied. In addition, the following condition holds.

(f4) $f(x,-u)=-f(x, u), \forall(x, u) \in \mathbb{R}^{N} \times \mathbb{R}$.

Then problem (1.1) possesses infinitely many nontrivial solutions.

It is easy to see that $(f 2)$ is satisfied if the following condition holds.

$\left(f 2^{\prime}\right)$ there exist an open set $\Omega \subset \mathbb{R}^{3}$ and three constants $\zeta, \eta>0, \kappa \in(1,2)$ such that

$$
u f(x, u) \geq \eta|u|^{\kappa}, \quad \forall(x, u) \in \Omega \times[-\zeta, \zeta] .
$$

Therefore, by Theorems 1.1 and 1.2, we have the following corollaries.

Corollary 1.1. In Theorem 1.1 and Theorem 1.3, if assumption $(f 2)$ is replayed by $\left(f 2^{\prime}\right)$, then the conclusions still hold.

Corollary 1.2. Suppose that $V$ satisfies $(V)$ and the following conditions hold. (f5) $F(x, u)=q(x) G(u)$, where $G \in C^{1}(\mathbb{R} \times \mathbb{R})$ and $q \in C\left(\mathbb{R}^{N} \times \mathbb{R}\right) \cap$ $L^{\frac{2}{2-\kappa_{1}}}\left(\mathbb{R}^{N} \times \mathbb{R}\right), \kappa_{1} \in(1,2)$ is a constant, such that $q\left(x_{0}\right)>0$ for some $x_{0} \in \mathbb{R}^{N}$;

(f6) There exist constants $m, M>0$ and $\kappa_{0} \in(1,2)$ such that

$$
m|u|^{\kappa_{0}} \leq G(u) \leq M|u|^{\kappa_{1}}, \quad \forall(x, u) \in \mathbb{R}^{N} \times \mathbb{R} .
$$

Then problem (1.1) possesses at least one nontrivial solution. 
Corollary 1.3. Suppose that all the conditions in Corollary 1.1 hold. In addition, the following condition satisfies.

(f7) $G(-u)=G(u), \forall u \in \mathbb{R}$.

Then problem (1.1) possesses infinitely many nontrivial solutions.

The sequel of this paper is organized as follows. In Section 2, some preliminary results are presented. We give the proof of our main results in Section 3. Finally, one example is given to illustrate our results.

\section{Preliminaries}

Throughout this paper, we work in the following Hilbert space

$$
E:=\left\{u \in H^{1}\left(\mathbb{R}^{N}\right): \int_{\mathbb{R}^{N}}\left[|\nabla u|^{2}+V(x)|u|^{2}\right] d x<+\infty\right\},
$$

which equipped with the inner product

$$
\langle u, v\rangle=\int_{\mathbb{R}^{N}}[\nabla u \nabla v+V(x) u v] d x, \quad u, v \in E
$$

and the associated norm

$$
\|u\|=\left(\int_{\mathbb{R}^{N}}\left[|\nabla u|^{2}+V(x) u^{2}\right] d x\right)^{\frac{1}{2}}, u \in E .
$$

As usual, for $1 \leq p<+\infty$, we let

$$
\|u\|_{p}=\left(\int_{\mathbb{R}^{N}}|u|^{p} d x\right)^{\frac{1}{p}}, u \in L^{p}\left(\mathbb{R}^{N}\right)
$$

and

$$
\|u\|_{\infty}=\operatorname{ess} \sup |u(x)|, u \in L^{\infty}\left(\mathbb{R}^{N}\right) .
$$

Evidently, $E$ is continuously embedded into $L^{p}\left(\mathbb{R}^{N}\right)$ for $2 \leq p \leq 2^{*}$ under the condition $(V)$, that is, there exists $\gamma_{p}>0$ such that

$$
\|u\|_{p} \leq \gamma_{p}\|u\|, \quad \forall u \in E, \quad p \in\left[2,2^{*}\right] .
$$

Definition 2.1. A function $u \in E$ is said to be a (weak) solution of (1.1) if for any $v \in E$, there holds

$$
a\langle u, v\rangle+b\|u\|^{2}\langle u, v\rangle=\int_{\mathbb{R}^{N}} f(x, u) v d x .
$$

Lemma 2.1. Assume that $(V)$ and $(f 1)$ hold. Then the functional $J(u): E \rightarrow$ $\mathbb{R}$ defined by

$$
J(u)=\frac{a}{2}\|u\|^{2}+\frac{b}{4}\|u\|^{4}-\int_{\mathbb{R}^{N}} F(x, u) d x
$$

is well defined and of class $C^{1}(E, \mathbb{R})$ and

$$
\left\langle J^{\prime}(u), v\right\rangle=a\langle u, v\rangle+b\|u\|^{2}\langle u, v\rangle-\int_{\mathbb{R}^{N}} f(x, u) v d x .
$$

Furthermore, the critical points of $J(u)$ in $E$ are the solutions of (1.1). 
Proof. It follows from $(f 1)$ that

$$
|F(x, u)| \leq \sum_{i=1}^{m} a_{i}(x)|u|^{\delta_{i}} .
$$

By $(V),(2.5)$ and the Hölder inequality, for any $u \in E$, we get that

$$
\begin{aligned}
\int_{\mathbb{R}^{N}}|F(x, u)| d x & \leq \sum_{i=1}^{m} \int_{\mathbb{R}^{N}} a_{i}(x)|u|^{\delta_{i}} d x \\
& \leq \sum_{i=1}^{m} \int_{\mathbb{R}^{N}}\left(\frac{V(x)}{\alpha}\right)^{\frac{\delta_{i}}{2}} a_{i}(x)|u|^{\delta_{i}} d x \\
& \leq \sum_{i=1}^{m} \alpha^{-\frac{\delta_{i}}{2}}\left\|a_{i}(x)\right\|_{\frac{2}{2-\delta_{i}}}\left(\int_{\mathbb{R}^{N}} V(x)|u|^{2} d x\right)^{\frac{\delta_{i}}{2}} \\
& \leq C_{1} \sum_{i=1}^{m}\|u\|^{\delta_{i}} .
\end{aligned}
$$

Thus, $J(u)$ is well defined on $E$ by (2.3) and (2.6).

Now, we show that (2.4) holds. By $(f 1)$, for any $u, v \in E, l \in(0,1)$, $\theta(x): \mathbb{R}^{N} \rightarrow(0,1)$ and the Hölder inequality, we obtain that

$$
\begin{aligned}
& \int_{\mathbb{R}^{N}} \max _{l \in(0,1)}|f(x, u(x)+l \theta(x) v(x)) v(x)| d x \\
= & \int_{\mathbb{R}^{N}} \max _{l \in(0,1)}|f(x, u(x)+l \theta(x) v(x)) \| v(x)| d x \\
\leq & \sum_{i=1}^{m} \delta_{i} \int_{\mathbb{R}^{N}} a_{i}(x)|u(x)+\theta(x) v(x)|^{\delta_{i}-1}|v(x)| d x \\
\leq & C_{2} \sum_{i=1}^{m}\left(\int_{\mathbb{R}^{N}}\left|a_{i}\right|^{\frac{2}{2-\delta_{i}}} d x\right)^{\frac{2-\delta_{i}}{2}}\left(\int_{\mathbb{R}^{N}} V(x)|u(x)|^{2} d x\right)^{\frac{\delta_{i}-1}{2}}\left(\int_{\mathbb{R}^{N}} V(x)|v(x)|^{2} d x\right)^{\frac{1}{2}} \\
& +C_{2} \sum_{i=1}^{m}\left(\int_{\mathbb{R}^{N}}\left|a_{i}\right|^{\frac{2}{2-\delta_{i}}} d x\right)^{\frac{2-\delta_{i}}{2}}\left(\int_{\mathbb{R}^{N}} V(x)|v(x)|^{2} d x\right)^{\frac{\delta_{i}}{2}} \\
\leq & C_{2} \sum_{i=1}^{m}\left\|a_{i}\right\|_{\frac{2}{2-\delta_{i}}}\left(\|u\|^{\delta_{i}-1}+\|v\|^{\delta_{i}-1}\right)\|v\|<+\infty .
\end{aligned}
$$

Then by (2.3), (2.7) and Lebesgue's Dominated Convergence Theorem, we have

$$
\begin{aligned}
\left\langle J^{\prime}(u), v\right\rangle & =\lim _{l \rightarrow 0^{+}} \frac{J(u+l v)-J(u)}{l} \\
& =\left[a+b\left(\int_{\mathbb{R}^{N}}\left(|\nabla u|^{2}+V(x)|u|^{2}\right) d x\right)\right] \int_{\mathbb{R}^{N}}[\nabla u \nabla v+V(x) u v] d x
\end{aligned}
$$




$$
\begin{aligned}
& -\lim _{l \rightarrow 0^{+}} \frac{1}{l} \int_{\mathbb{R}^{N}}[F(x, u(x)+l v(x))-F(x, u(x))] d x \\
= & {\left[a+b\left(\int_{\mathbb{R}^{N}}\left(|\nabla u|^{2}+V(x)|u|^{2}\right) d x\right)\right] \int_{\mathbb{R}^{N}}[\nabla u \nabla v+V(x) u v] d x } \\
& -\lim _{l \rightarrow 0^{+}} \int_{\mathbb{R}^{N}} f(x, u(x)+\theta(x) l v(x)) v(x) d x \\
= & {\left[a+b\left(\int_{\mathbb{R}^{N}}\left(|\nabla u|^{2}+V(x)|u|^{2}\right) d x\right)\right] \int_{\mathbb{R}^{N}}[\nabla u \nabla v+V(x) u v] d x } \\
& -\int_{\mathbb{R}^{N}} f(x, u(x)) v(x) d x,
\end{aligned}
$$

which implies that (2.4) holds. Moreover, by a standard argument, it is easy to show that the critical points of $J(u)$ in $E$ are the solutions of problem (1.1), see for example [3].

In what follows, we show that $J^{\prime}(u)$ is continuous. According to (2.3), it suffices to show that

$$
\Phi^{\prime} \triangleq \int_{\mathbb{R}^{N}} f(x, u) v d x
$$

is continuous. Let $u_{k} \rightarrow u$ in $E$. Then

$$
u_{k} \rightarrow u, \quad \text { in } L^{2}\left(\mathbb{R}^{N}\right), \quad u_{k} \rightarrow u \quad \text { a.e. in } \mathbb{R}^{N} .
$$

We show that

$$
\int_{\mathbb{R}^{N}}\left|f\left(x, u_{k}\right)-f(x, u)\right|^{2} d x \rightarrow 0, \quad \text { as } \quad k \rightarrow+\infty .
$$

To prove (2.10), arguing by contradiction, suppose that there exists a constant $\varepsilon_{0}>0$ and a subsequence (also denotes $\left\{u_{k}\right\}$ ) such that

$$
\int_{\mathbb{R}^{N}}\left|f\left(x, u_{k}\right)-f(x, u)\right|^{2} d x \geq \varepsilon_{0}, \quad \text { as } \quad k \rightarrow+\infty .
$$

Since $u_{k} \rightarrow u$ in $L^{2}\left(\mathbb{R}^{N}\right)$, passing to a subsequence if necessary we can assume that $\sum_{i=1}^{\infty}\left\|u_{k}-u\right\|_{2}^{2}<+\infty$. Set $w(x)=\left[\sum_{i=1}^{\infty}\left|u_{k}-u\right|^{2}\right]^{\frac{1}{2}}, x \in \mathbb{R}^{N}$, then $w(x) \in L^{2}\left(\mathbb{R}^{N}\right)$. For all $k \in \mathbb{N}$ and by $(f 1)$, we have

$$
\begin{aligned}
\left|f\left(x, u_{k}(x)\right)-f(x, u(x))\right|^{2} & \leq 2\left|f\left(x, u_{k}(x)\right)\right|^{2}+2|f(x, u(x))|^{2} \\
& \leq C_{3} \sum_{i=1}^{m}\left|a_{i}(x)\right|^{2}\left[\left|u_{k}(x)\right|^{2 \delta_{i}-2}+|u(x)|^{2 \delta_{i}-2}\right] \\
& \leq C_{3} \sum_{i=1}^{m}\left|a_{i}(x)\right|^{2}\left[|w(x)|^{2 \delta_{i}-2}+|u(x)|^{2 \delta_{i}-2}\right] \\
& :=g(x), \quad \text { a.e. in } \mathbb{R}^{N}
\end{aligned}
$$


and

$$
\begin{aligned}
\int_{\mathbb{R}^{N}} g(x) d x & =C_{3} \sum_{i=1}^{m} \int_{\mathbb{R}^{N}}\left|a_{i}(x)\right|^{2}\left[|w(x)|^{2 \delta_{i}-2}+|u(x)|^{2 \delta_{i}-2}\right] d x \\
& \leq C_{3} \sum_{i=1}^{m}\left\|a_{i}(x)\right\|_{\frac{2}{2-\delta_{i}}}^{2}\left[\|w(x)\|_{2}^{2 \delta_{i}-2}+\|u(x)\|_{2}^{2 \delta_{i}-2}\right]<+\infty .
\end{aligned}
$$

Then by (2.9), (2.12), (2.13) and Lebesgue's dominated convergence theorem, we have

$$
\int_{\mathbb{R}^{N}}\left|f\left(x, u_{k}\right)-f(x, u)\right|^{2} d x \rightarrow 0, \quad \text { as } \quad k \rightarrow+\infty,
$$

which contradicts (2.11). So, (2.10) holds.

By (2.8), (2.14) and the Hölder inequality, for all given $v \in E$, we have

$$
\begin{aligned}
\left|\left\langle\Phi^{\prime}\left(u_{k}\right)-\Phi^{\prime}(u), v\right\rangle\right| & =\left|\int_{\mathbb{R}^{N}}\left[f\left(x, u_{k}\right)-f(x, u)\right] v d x\right| \\
& \leq C_{4}\|v\|\left(\int_{\mathbb{R}^{N}}\left|f\left(x, u_{k}\right)-f(x, u)\right|^{2} d x\right)^{\frac{1}{2}} \rightarrow 0,
\end{aligned}
$$

as $k \rightarrow+\infty$, which implies the continuity of $\Phi^{\prime}$. Hence, $J \in C^{1}(E, \mathbb{R})$.

In what follows, we collect some definitions and propositions which are very useful throughout the present paper and we will use in the next section.

Let $E$ be a Banach space, $J(u) \in C^{1}(E, \mathbb{R})$ and $c \in \mathbb{R}$. Set

$\Sigma=\{A \subset E-\{0\}: \mathrm{A}$ is closed in $\mathrm{E}$ and symmetric with respect to 0$\}$,

$$
K_{c}=\left\{u \in E: J(u)=c, J^{\prime}(u)=0\right\}, \quad J^{c}=\{u \in E: J(u) \leq c\} .
$$

Definition 2.2 (Chang [4]). Let $u$ be an isolated critical point of $J$ with $J(u)=c$, for $c \in \mathbb{R}$, and let $U$ be a neighborhood of $u$, containing the unique critical point. We call

$$
C_{q}(J, u):=H_{q}\left(J^{c} \cap U, J^{c} \cap U \backslash\{u\}\right), \quad q=0,1,2, \ldots,
$$

the $q$ th critical group of $J$ at $u$, where $J^{c}:=\{u \in E: J(u) \leq c\}, H_{q}(\cdot, \cdot)$ stands for the $q$ th singular relative homology group with integer coefficients.

We say that $u$ is a homological nontrivial critical point of $J$ if at least one of its critical groups is nontrivial.

Proposition 2.1 (Bartsch and Liu [2]). Let 0 be a critical point of $J$ with $J(0)=0$. Assume that $J$ has a local linking at 0 with respect to $E=E_{1} \bigoplus E_{2}$, $k=\operatorname{dim} E_{1}<\infty$, that is, there exists $\rho>0$ small such that

$$
J(u) \leq 0, \quad u \in E_{1},\|u\| \leq \rho \quad \text { and } \quad J(u)>0, \quad u \in E_{2}, 0<\|u\| \leq \rho .
$$

Then $C_{k}(J, 0) \not 0$, that is, 0 is a homological nontrivial critical point of $J$. 
Definition 2.3 ([19]). For $A \in \Sigma$, we say genus of $A$ is $n$ (denoted by $\gamma(A)=n$ ) if there is an odd map $\varphi \in C\left(A, \mathbb{R}^{N} \backslash\{0\}\right)$ and $n$ is the smallest integer with this property.

We say that $J \in C^{1}(E, \mathbb{R})$ satisfies $(P S)$-condition if any sequence $\left\{u_{n}\right\}$ in E such that

$$
J\left(u_{n}\right) \rightarrow c, \quad J^{\prime}\left(u_{n}\right) \rightarrow 0 \quad \text { as } \quad n \rightarrow \infty
$$

has a convergent subsequence.

Proposition 2.2. Let $E$ be a real Banach space and $J \in C^{1}(E, \mathbb{R})$ satisfy the $(P S)$-condition. If $J$ is bounded from below, then $c=\inf _{E} J$ is a critical value of $J$.

Proposition 2.3 (Zhang and $\mathrm{Li}[25]$ ). Assume that J satisfies the (PS)condition and is bounded from below. If $J$ has a critical point that is homological nontrivial and is not the minimizer of J. Then $J$ has at least three critical points.

Proposition 2.4 ([20]). Let $J$ be an even $C^{1}$ functional on $E$ and satisfy the $(P S)$-condition. For any $n \in \mathbb{N}$, set

$$
\Sigma_{n}=\{A \in \Sigma: \gamma(A) \geq n\}, \quad c_{n}=\inf _{A \in \Sigma_{n}} \sup _{u \in A} J(u) .
$$

(1) If $\Sigma_{n} \neq \emptyset$ and $c_{n} \in \mathbb{R}$, then $c_{n}$ is a critical value of $J$;

(2) If there exists $r \in \mathbb{N}$ such that $c_{n}=c_{n+1}=\cdots=c_{n+r}=c \in \mathbb{R}$, and $c \neq J(0)$, then $\gamma\left(K_{c}\right) \geq r+1$.

\section{Proof of main results}

We begin this section by verifying the follow compactness lemma which shows that the functional $J$ satisfies $(P S)$-condition and is bounded from below.

Lemma 3.1. Assume that the conditions $(V)$ and $(f 1)$ hold, then $J$ is bounded from below and satisfies the (PS)-condition.

Proof. First, we show that $J$ is bounded from below. By $(2.3),(f 1)$ and the Hölder inequality, one has

$$
\begin{aligned}
J(u) & =\frac{a}{2}\|u\|^{2}+\frac{b}{4}\|u\|^{4}-\int_{\mathbb{R}^{N}} F(x, u) d x \\
& \geq \frac{a}{2}\|u\|^{2}+\frac{b}{4}\|u\|^{4}-\sum_{i=1}^{m} \int_{\mathbb{R}^{N}} a_{i}(x)|u|^{\delta_{i}} d x \\
& \geq \frac{a}{2}\|u\|^{2}+\frac{b}{4}\|u\|^{4}-C_{5} \sum_{i=1}^{m}\left(\int_{\mathbb{R}^{N}}\left|a_{i}(x)\right|^{\frac{2}{2-\delta_{i}}} d x\right)^{\frac{2-\delta_{i}}{2}}\left(\int_{\mathbb{R}^{N}} V(x)|u|^{2} d x\right)^{\frac{\delta_{i}}{2}}
\end{aligned}
$$




$$
\geq \frac{a}{2}\|u\|^{2}+\frac{b}{4}\|u\|^{4}-C_{5} \sum_{i=1}^{m}\left\|a_{i}\right\|_{\frac{2}{2-\delta_{i}}}\|u\|^{\delta_{i}} .
$$

Since $1<\delta_{i}<2(i=1,2, \ldots, m),(3.1)$ implies that $J(u) \rightarrow+\infty$ as $\|u\| \rightarrow+\infty$. Consequently, $J$ is bounded from below.

Next, we prove that $J$ satisfies the $(P S)$-condition. Assume that $\left\{u_{n}\right\}_{n \in \mathbb{N}} \subset$ $E$ is a sequence such that (2.15) holds. Then by (3.1), there exists a constant $M>0$ such that

$$
\left\|u_{n}\right\| \leq M, \quad \forall n \in \mathbb{N} .
$$

Going if necessary to a subsequence we can assume that $u_{n} \rightarrow u_{0}$ in $E$. Hence, by Rellich embedding theorem, we have

$$
u_{n} \rightarrow u_{0}, \text { in } L_{l o c}^{s}\left(\mathbb{R}^{N}\right), \quad s \in\left[2,2^{*}\right) .
$$

By $(f 1)$, for any given $\varepsilon>0$, we can choose $\rho>0$ such that

$$
\left(\int_{|x| \geq \rho}\left|a_{i}\right|^{\frac{2}{2-\delta_{i}}} d x\right)^{\frac{2-\delta_{i}}{2}}<\varepsilon .
$$

It follows from (2.12), (2.13), (3.2), (3.3) and the Hölder inequality that there exists $n_{0} \in \mathbb{N}$ such that

$$
\begin{aligned}
& \int_{|x| \leq \rho}\left|f\left(x, u_{n}\right)-f\left(x, u_{0}\right)\right|\left|u_{n}-u_{0}\right| d x \\
\leq & \left(\int_{|x| \leq \rho}\left|f\left(x, u_{n}\right)-f\left(x, u_{0}\right)\right|^{2} d x\right)^{\frac{1}{2}}\left(\int_{|x| \leq \rho}\left|u_{n}-u_{0}\right|^{2} d x\right)^{\frac{1}{2}} \\
\leq & \varepsilon\left(\int_{|x| \leq \rho}\left|f\left(x, u_{n}\right)-f\left(x, u_{0}\right)\right|^{2} d x\right)^{\frac{1}{2}} \\
\leq & C_{6} \varepsilon\left[\sum_{i=1}^{m}\left(\left\|u_{n}\right\|_{2}^{2 \delta_{i}-2}+\left\|u_{0}\right\|_{2}^{2 \delta_{i}-2}\right)\right]^{\frac{1}{2}} \\
\leq & C_{7} \varepsilon\left[\sum_{i=1}^{m}\left(M^{2 \delta_{i}-2}+\left\|u_{0}\right\|_{2}^{2 \delta_{i}-2}\right)\right]^{\frac{1}{2}} \\
\leq & C_{8} \varepsilon
\end{aligned}
$$

for $n \geq n_{0}$.

On the other hand, it follows from (2.1), (3.4) and the Hölder inequality that

$$
\begin{aligned}
& \int_{|x|>\rho}\left|f\left(x, u_{n}\right)-f\left(x, u_{0}\right)\right|\left|u_{n}-u_{0}\right| d x \\
\leq & \int_{|x|>\rho}\left[\left|f\left(x, u_{n}\right)\right|+\left|f\left(x, u_{0}\right)\right|\right]\left(\left|u_{n}-u_{0}\right|\right) d x
\end{aligned}
$$




$$
\begin{aligned}
& \leq \sum_{i=1}^{m} \delta_{i} \int_{|x|>\rho}\left|a_{i}(x)\right|\left[\left|u_{n}\right|^{\delta_{i}}+\left|u_{0}\right|^{\delta_{i}}+\left|u_{n}\right|^{\delta_{i}-1}\left|u_{0}\right|+\left|u_{0}\right|^{\delta_{i}-1}\left|u_{n}\right|\right] d x \\
& \leq \varepsilon \sum_{i=1}^{m} \delta_{i}\left(\left\|u_{n}\right\|_{2}^{\delta_{i}}+\left\|u_{0}\right\|_{2}^{\delta_{i}}+\left\|u_{n}\right\|_{2}^{\delta_{i}-1}\left\|u_{0}\right\|_{2}+\left\|u_{0}\right\|_{2}^{\delta_{i}-1}\left\|u_{n}\right\|_{2}\right) \\
& \leq C_{9} \varepsilon .
\end{aligned}
$$

Since $\varepsilon$ is arbitrary, combining (3.5) and (3.6) we get

$$
\int_{\mathbb{R}^{N}}\left|f\left(x, u_{n}\right)-f\left(x, u_{0}\right)\right|\left|u_{n}-u_{0}\right| d x \rightarrow 0 \quad \text { as } \quad n \rightarrow+\infty .
$$

It follows from (2.4) that

$$
\begin{aligned}
& \min \left\{b\left\|u_{n}\right\|^{2}+a, b\left\|u_{0}\right\|^{2}+a\right\}\left\|u_{n}-u_{0}\right\|^{2} \\
\leq & \left\langle J^{\prime}\left(u_{n}\right)-J^{\prime}\left(u_{0}\right), u_{n}-u_{0}\right\rangle+\int_{\mathbb{R}^{N}}\left[f\left(x, u_{n}\right)-f\left(x, u_{0}\right)\right]\left(u_{n}-u_{0}\right) d x .
\end{aligned}
$$

Obviously,

$$
\left\langle J^{\prime}\left(u_{n}\right)-J^{\prime}\left(u_{0}\right), u_{n}-u_{0}\right\rangle \rightarrow 0 \quad \text { as } n \rightarrow+\infty .
$$

Combining (3.7), (3.8) and (3.9), we obtain that $u_{n} \rightarrow u_{0}$ in $E$. Hence, $J$ satisfies $(P S)$-condition. The proof is complete.

Now, we are in the position to give the proofs of Theorem 1.1, Theorem 1.2 and Theorem 1.3.

Proof of Theorem 1.1. In view of Lemma 2.1, $J \in C^{1}(E, \mathbb{R})$. By Lemma 3.1 and Proposition 2.1, we obtain $c=\inf _{E} J(u)$ is a critical value of $J$, that is, there exists a critical point $u^{*} \in E$ such that $J\left(u^{*}\right)=c$.

Now, we show that $u^{*} \neq 0$. Let $u_{0} \in\left(W_{0}^{1,2}(\Omega) \cap E\right) \backslash\{0\}$. Then by (2.3) and $(f 2)$, we infer that

$$
\begin{aligned}
J\left(t u_{0}\right) & =\frac{a t^{2}}{2}\left\|u_{0}\right\|^{2}+\frac{\lambda t^{4}}{4}\left\|u_{0}\right\|^{4}-\int_{\mathbb{R}^{N}} F\left(x, t u_{0}\right) d x \\
& \leq \frac{a t^{2}}{2}\left\|u_{0}\right\|^{2}+\frac{\lambda t^{4}}{4}\left\|u_{0}\right\|^{4}-\eta t^{\kappa} \int_{\Omega}\left|u_{0}(x)\right|^{\kappa} d x .
\end{aligned}
$$

Since $1<\kappa<2$, it follows from (3.10) that $J\left(t u_{0}\right)<0$ for $t>0$ small enough. Thus, we get that $J\left(u^{*}\right)=c<0$. Therefore, $u^{*}$ is a nontrivial critical point of $J$ with $J\left(u^{*}\right)=\inf _{E} J(u)$ and is a nontrivial solution of problem (1.1). The proof is complete.

Proof of Theorem 1.2. Lemma 3.1 shows that $J$ satisfies $(P S)$ condition and is bounded from below. From (2.4) and the definition of $F(x, u)$, it is easy to check that $J(0)=0$. In what follows, we show that 0 is a homological nontrivial critical point of $J$. Since $E$ is a Hilbert space, we choose an orthogonal basis $\left\{e_{j}\right\}$ of $E$ and let $E=E^{-} \bigoplus E^{+}$, where

$$
E^{-}:=\operatorname{span}\left\{e_{1}, \ldots, e_{k}\right\} \quad \text { and } \quad E^{+}:=\left(E^{-}\right)^{\perp} .
$$


On one hand, for $u \in E^{-}$, we have from (2.4) and (f3) that

$$
\begin{aligned}
J(u) & =\frac{a}{2}\|u\|^{2}+\frac{b}{4}\|u\|^{4}-\int_{\mathbb{R}^{N}} F(x, u) d x \\
& \leq \frac{a}{2}\|u\|^{2}+\frac{b}{4}\|u\|^{4}-c_{1} \int_{\mathbb{R}^{N}} u^{\tau} d x \\
& \leq \frac{a}{2}\|u\|^{2}+\frac{b}{4}\|u\|^{4}-c_{1}\|u\|_{\tau}^{\tau} .
\end{aligned}
$$

Sine all norms on a finite dimensional space are equivalent, we deduce from (3.12) that

$$
J(u) \leq \frac{a}{2}\|u\|^{2}+\frac{b}{4}\|u\|^{4}-c_{1}\|u\|^{\tau},
$$

which implies that $J(u) \leq 0$ if we choose $\|u\|$ small enough since $1<\tau<2$.

On the other hand, for $u \in E^{+}$, from (2.1), (2.4) and (f3) we have

$$
\begin{aligned}
J(u) & =\frac{a}{2}\|u\|^{2}+\frac{b}{4}\|u\|^{4}-\int_{\mathbb{R}^{N}} F(x, u) d x \\
& \geq \frac{a}{2}\|u\|^{2}+\frac{b}{4}\|u\|^{4}-c_{2} \int_{\mathbb{R}^{N}} u^{2} d x \\
& \geq \frac{a}{2}\|u\|^{2}+\frac{b}{4}\|u\|^{4}-c_{2} \gamma_{2}^{2}\|u\|^{2} \\
& \geq C_{9}\|u\|^{2}+\frac{b}{4}\|u\|^{4}
\end{aligned}
$$

which means that $J(u) \leq 0$ if we choose $\|u\|$ small enough.

From (2.4) and the definition of $E^{-}$, it is easy to see that $J(0)=0$ and $k=\operatorname{dim} E^{-}<\infty$. Therefore, combining the above arguments and Proposition 2.1 , we have $C_{k}(J, 0) \nsubseteq 0$. This means that 0 is an homological nontrivial critical point of $J$. Moreover, (3.12) shows that 0 is not the minimizer of $J$. Thus all the conditions of Proposition 2.3 are satisfied and we get that the problem (1.1) has two nontrivial solutions. We complete the proof.

Proof of Theorem 1.3. By Lemma 2.1 and Lemma 3.1, we get that $J \in C^{1}(E$, $\mathbb{R})$ is bounded from below and satisfies the $(P S)$-condition. It follows from (2.3) and $(f 3)$ that $J$ is even and $J(0)=0$.

Now, we prove that for any $n \in \mathbb{N}$ there exists $\varepsilon>0$ such that

$$
\gamma\left(J^{-\varepsilon}\right) \geq n \text {. }
$$

For any $n \in \mathbb{N}$, we take $n$ disjoint open sets $\Omega_{i}$ such that

$$
\bigcup_{i=1}^{n} \Omega_{i} \subset \Omega .
$$

For each $i \in\{1,2, \ldots, n\}$, let $u_{i} \in\left(W_{0}^{1,2}\left(\Omega_{i}\right) \cap E\right) \backslash\{0\}$ and $\left\|u_{i}\right\|=1$, and

$$
E_{n}=\operatorname{span}\left\{u_{1}, u_{2}, \ldots, u_{n}\right\}, \quad S_{n}=\left\{u \in E_{n}:\|u\|=1\right\} .
$$


Thus, for any $u \in E_{n}$, there exist $\lambda_{i} \in \mathbb{R}, i=1,2, \ldots, n$ such that

$$
u(x)=\sum_{i=1}^{n} \lambda_{i} u_{i} \quad \text { for } x \in \mathbb{R}^{N}
$$

Then we have

$$
\|u\|_{\kappa}=\left(\int_{\mathbb{R}^{N}}|u(x)|^{\kappa} d x\right)^{\frac{1}{\kappa}}=\left(\sum_{i=1}^{n}\left|\lambda_{i}\right|^{\kappa} \int_{\Omega_{i}}\left|u_{i}(x)\right|^{\kappa} d x\right)^{\frac{1}{\kappa}}
$$

and

$$
\begin{aligned}
\|u\|^{2} & =\int_{\mathbb{R}^{N}}\left[|\nabla u(x)|^{2}+V(x)|u(x)|^{2}\right] d x \\
& =\sum_{i=1}^{n} \lambda_{i}^{2} \int_{\Omega_{i}}\left[\left|\nabla u_{i}(x)\right|^{2}+V(x)\left|u_{i}(x)\right|^{2}\right] d x \\
& =\sum_{i=1}^{n} \lambda_{i}^{2} \int_{\mathbb{R}^{N}}\left[\left|\nabla u_{i}(x)\right|^{2}+V(x)\left|u_{i}(x)\right|^{2}\right] d x \\
& =\sum_{i=1}^{n} \lambda_{i}^{2}\left\|u_{i}(x)\right\|^{2} \\
& =\sum_{i=1}^{n} \lambda_{i}^{2} .
\end{aligned}
$$

Since $E_{n}$ is a finite dimensional normed space, one gets that all norms of $E_{n}$ are equivalent. Therefore, there is a constant $\vartheta>0$ such that

$$
\vartheta\|u\| \leq\|u\|_{\kappa} \text { for } u \in E_{n} .
$$

By $(f 3),(3.16),(3.17)$ and (3.18), we have

$$
\begin{aligned}
J(t u) & =\frac{a t^{2}}{2}\|u\|^{2}+\frac{\lambda t^{4}}{4}\|u\|^{4}-\int_{\mathbb{R}^{N}} F(x, t u) d x \\
& =\frac{a t^{2}}{2}\|u\|^{2}+\frac{\lambda t^{4}}{4}\|u\|^{4}-\sum_{i=1}^{n} \int_{\Omega_{i}} F\left(x, t \lambda_{i} u_{i}(x)\right) d x \\
& \leq \frac{a t^{2}}{2}\|u\|^{2}+\frac{\lambda t^{4}}{4}\|u\|^{4}-\eta t^{\kappa} \sum_{i=1}^{n}\left|\lambda_{i}\right|^{\kappa} \int_{\Omega_{i}}\left|u_{i}(x)\right|^{\kappa} d x \\
& \leq \frac{a t^{2}}{2}\|u\|^{2}+\frac{\lambda t^{4}}{4}\|u\|^{4}-\eta t^{\kappa}\|u\|_{\kappa}^{\kappa} \\
& \leq \frac{a t^{2}}{2}\|u\|^{2}+\frac{\lambda t^{4}}{4}\|u\|^{4}-\eta \vartheta^{\kappa} t^{\kappa}\|u\|^{\kappa} \\
& =\frac{a}{2} t^{2}+\frac{\lambda}{4} t^{4}-\eta \vartheta^{\kappa} t^{\kappa}, \quad \forall u \in S_{n} .
\end{aligned}
$$


So for $t$ small enough, (3.19) implies that there exist $\varepsilon>0$ and $\tau>0$ such that

$$
J(\tau u)<-\varepsilon \text { for } u \in S_{n} .
$$

Let

$$
S_{n}^{\tau}=\left\{\tau u: u \in S_{n}\right\}, \quad Q=\left\{\left(\lambda_{1}, \lambda_{2}, \ldots, \lambda_{n}\right): \sum_{i=1}^{n} \lambda_{i}^{2}<\tau^{2}\right\} .
$$

It follows from (3.20) that $J(u)<-\varepsilon$ for $u \in S_{n}^{\tau}$, which, together with the fact that $J \in C^{1}(E, \mathbb{R})$ and $J$ is even, implies that

$$
S_{n}^{\tau} \subset J^{-\varepsilon} \in \Sigma \text {. }
$$

On the other hand, from (3.15) and (3.17), there exits an odd homeomorphism mapping $\psi \in C\left(S_{n}^{\tau}, \partial Q\right)$. By the monotonicity of genus and Proposition 7.7 in [19], we deduce

$$
\gamma\left(J^{-\varepsilon}\right) \geq \gamma\left(S_{n}^{\tau}\right)=n
$$

Thus, (3.14) holds.

Set

$$
c_{n}=\inf _{A \in \Sigma_{n}} \sup _{u \in A} J(u) .
$$

It follows from (3.21) and the fact that $J$ is bounded from below on $E$ that $-\infty<c_{n} \leq-\varepsilon<0$, that is, for any $n \in \mathbb{N}, c_{n}$ is a real negative number.

Therefore, $J$ has infinitely many nontrivial critical points by Proposition 2.2. So, problem (1.1) possesses infinitely many nontrivial solutions.

\section{Example}

In this section, an example is given to illustrate our main results.

Example 4.1. In problem (1.1), let $V(x)=3+\sin |x|^{2}$ and let $\alpha=1$. Then, condition $(V)$ satisfies. For $\forall(x, u) \in \mathbb{R}^{N} \times \mathbb{R}$, let

$$
f(x, u)=\frac{1+\sin ^{2}|x|}{1+|x|^{\frac{3}{2}}}|u|^{\frac{-3}{4}} u+\frac{4}{3 e^{|x|}}|u|^{\frac{-2}{3}} u+\frac{3 \cos |x|}{2 e^{3}|x|}|u|^{\frac{-1}{2}} u .
$$

Clearly,

and

$$
|f(x, u)| \leq \frac{2}{1+|x|^{\frac{3}{2}}}|u|^{\frac{1}{4}}+\frac{4}{3 e^{|x|}}|u|^{\frac{1}{3}}+\frac{3}{2 e^{3}|x|}|u|^{\frac{1}{2}}
$$

$$
\begin{aligned}
F(x, u) & =\frac{1+\sin ^{2}|x|}{1+|x|^{\frac{3}{2}}} \frac{4}{5}|u|^{\frac{5}{4}} u+\frac{4}{e^{|x|}}|u|^{\frac{4}{3}}+\frac{\cos |x|}{e^{3}|x|}|u|^{\frac{3}{2}} \\
& \geq \frac{1}{e^{|x|}}|u|^{\frac{4}{3}}, \quad \forall(x, u) \in B_{\frac{\pi}{2}} \times[-1,1] .
\end{aligned}
$$

Here, we can choose $a$ large enough such that $0<c_{1}<c_{2}<\frac{a}{\varsigma \gamma_{2}^{2}}$ holds, where $c_{1}=\eta=\frac{1}{e^{|x|}}, c_{2}=6 \max \left\{a_{1}, a_{2}, a_{3}\right\}$. Thus $(f 1),(f 2),(f 3)$ and $(f 4)$ are satisfied with

$$
\delta_{1}=\frac{1}{4}, \quad \delta_{2}=\frac{1}{3}, \quad \delta_{3}=\frac{1}{2},
$$




$$
\begin{gathered}
a_{1}=\frac{8}{5\left(1+|x|^{\frac{3}{2}}\right)}, \quad a_{2}=\frac{4}{3 e^{|x|}}, \quad a_{3}=\frac{3}{2 e^{3}|x|}, \\
\zeta=1, \quad \Omega=B_{\frac{\pi}{2}}, \quad \eta=\frac{1}{e^{|x|}}, \quad \kappa=\frac{4}{3} .
\end{gathered}
$$

By Theorem 1.1, Theorem 1.2 and Theorem 1.3, problem (1.1) has at leat one, two and infinitely many nontrivial solutions.

\section{References}

[1] C. O. Alves, F. J. S. A. Corrèa, and T. F. Ma, Positive solutions for a quasilinear elliptic equation of Kirchhoff type, Comput. Math. Appl. 49 (2005), no. 1, 85-93.

[2] T. Bartsch and S. Li, Critical point theory for asymptotically quadratic functionals and applications to problems with resonance, Nonlinear Anal. 28 (1997), no. 3, 419-441.

[3] V. Benci and D. Fortunato, An eigenvalue problem for the Schrödinger-Maxwell equations, Topol. Methods Nonlinear Anal. 11 (1998), no. 2, 283-293.

[4] K. Chang, Infinite Dimensional Morse Theory and Multiple Solution Problems, Birkhäuser, Boston, Basel, Berlin, 1993.

[5] P. Chen and C. Tian, Infinitely many solutions for Schröginger-Maxwell equations with indefinite sign subquadratic potentials, Appl. Math. Comput. 226 (2014), 492-502.

[6] B. Cheng and X. Wu, Existence results of positive solutions of Kirchhoff type problems, Nonlinear Anal. 71 (2009), no. 8, 4883-4892.

[7] L. Duan and L. Huang, Infinitely many solutions for sublinear Schröginger-Kirchhofftype equations with general potentials, Results Math. 66 (2014), no. 1-2, 181-197.

[8] X. He and W. Zou, Infinitely many positive solutions for Kirchhoff-type problems, Nonlinear Anal. 70 (2009), no. 3, 1407-1414.

[9] Existence and concentration behavior of positive solutions for a Kirchhoff equations in $\mathbb{R}^{3}$, J. Differential Equations 252 (2012), no. 2, 1813-1834.

[10] G. Kirchhoff, Mechanik, Teubner, Leipzig, 1883.

[11] G. Li and H. Ye, Existence of positive ground state solutions for the nonlinear Kirchhoff type equations in $\mathbb{R}^{3}$, J. Differential Equations 257 (2014), no. 2, 566-600.

[12] Y. Li, F. Li, and J. Shi, Existence of a positive solution to Kirchhoff type problems without compactness conditions, J. Differential Equations 253 (2012), no. 7, 2285-2294.

[13] J. L. Lions, On some questions in boundary value problems of mathematical physics, in: Contemporary Developments in Continum Mechanics and Partial Differential Equations, Proc. Internat. Sympos., Inst. Mat., Univ. Fed. Rio de Janeiro, Rio de Janeiro, 1977, in: North-Holland Math. Stud., vol. 30, North-Holland, Amsterdam, 1978, pp. 284-346. Invent. Math. 108 (1992), 247-262.

[14] W. Liu and X. He, Multiplicity of high energy solutions for superlinear Kirchhoff equations, J. Appl. Math. Comput. 39 (2012), no. 1-2, 473-487.

[15] Z. Liu, S. Guo, and Z. Zhang, Existence and multiplicity of solutions for a class of sublinear Schrödinger-Maxwell equations, Taiwanese J. Math. 17 (2013), no. 3, 857872 .

[16] A. Mao and Z. Zhang, Sign-changing and multiple solutions of Kirchhoff type problems without the P.S. condition, Nonlinear Anal. 70 (2009), no. 3, 1275-1287.

[17] J. Mawhin and M. Willem, Critical point theory and Hamiltonian systems, Springer, Berlin, 1989.

[18] K. Perera and Z. Zhang, Nontrivial solutions of Kirchhoff-type problems via the Yang index, J. Differential Equations 221 (2006), no. 1, 246-255.

[19] P. Rabinowitz, Minimax methods in critical point theory with applications to differential equations, in: CBMS Reg. Conf. Ser. in Math., Vol. 65, Amer. Math. Soc., Providence, RI, 1986. 
[20] A. Salvatore, Homoclinic orbits for a special class of nonautonomous Hamiltonian systems, in: Proceedings of the Second World Congress of Nonlinear Analysis, Part 8 (Athens, 1996), Nonlinear Anal. 30 (1997), no. 8, 4849-4857.

[21] J. Su and L. Zhao, An elliptic resonance problem with multiple solutions, J. Math. Anal. Appl. 319 (2006), no. 2, 604-616.

[22] J. Sun and T. Wu, Ground state solutions for an indefinite Kirchhoff type problem with steep potential well, J. Differential Equations 256 (2014), no. 4, 1771-1792.

[23] X. Wu, Existence of nontrivial solutions and high energy solutions for SchrödingerKirchhoff-type equations in $\mathbb{R}^{N}$, Nonlinear Anal. Real World Appl. 12 (2011), no. 2, $1278-1287$.

[24] High energy solutions of systems of Kirchhoff-type equations in $\mathbb{R}^{N}$, J. Math. Phys. 53 (2012), no. 6, 1-18.

[25] J. Zhang and S. Li, Multiple nontrivial solutions for some fourth-order semilinear elliptic problems, Nonlinear Anal. 60 (2005), no. 2, 221-230.

Haibo Chen

School of Mathematics and Statistics

Central South University

Changsha, Hunan 410083, P. R. China

E-mail address: math_chb@csu.edu.cn

HoNGLiAng LiU

School of Mathematics and Statistics

Central South University

Changsha, Hunan 410083, P. R. China

E-mail address: hongliangdl@163.com

LIPING XU

School of Mathematics and Statistics

Central South University

Changsha, Hunan 410083, P. R. China

AND

Department of Mathematics and Statistics

Henan University of Science and Technology

LuOYANG 471003, P. R. China

E-mail address: x.liping@126.com 